\title{
Sensitivity analysis and its application for dynamic improvement
}

\author{
NOBUYUKI OKUBO and TAKESHI TOI
}

\author{
Department of Precision Mechanics, Chuo University, Tokyo, Japan \\ e-mail: okubo@mech.chuo-u.ac.jp
}

\begin{abstract}
In order to determine appropriate points where natural frequency or mode shape under consideration can be effectively modified by structural modification, the theoretical background of sensitivity analysis is described, followed by experimental verification with a simple structure. Then it can be extended to (1) operational deflection shape, where a problem arises at offresonance frequency, (2) relative motion, (3) transmitted force through connecting points between components, (4) servo system, which consists of electrical elements with mechanical elements, and (5) acoustics generated by the vibration of the structure. To illusrate its effectiveness, many actual examples are given.
\end{abstract}

Keywords. Sensitivity analysis; dynamic improvement structural modification; laser beam printer; motorbike; disc drive; mechatronics; automobile engine.

\section{Introduction}

In order to solve the dynamic problem of a structure, the responses of the structure under operating conditions in terms of acceleration, acoustic pressure etc. should be evaluated at the beginning to define the problem, for example, the frequency range to be taken into account and so on. Modal analysis can then be used to model the relation between inputs and responses, such as a system model like frequency response functions (FRFs) in measurement. Based on the system model, together with the responses under operating conditions, the force identification inputs can be obtained.

By making use of structural modification methods to modify the system model with respect to the design parameter changes such as mass, stiffness or damping addition, one can predict the change of responses against the identified inputs, which are assumed to be independent of the modification. In order to achieve satisfactory dynamic improvement, the part of the structure to be modified has to be determined by iteratively perturbing the design parameters. This is, in practice, very difficult in the case of complicated structures where a number of design parameters are under consideration.

Therefore, sensitivity analysis plays an important role in determining which part of the structure should be modified for effective improvement. This is turn means that sensitivity 
analysis can provide the ratio of the change of modal parameters such as natural frequency, mode shape etc. to that of a design parameter such as mass, stiffness or damping.

In this paper, the theoretical background of sensitivity analysis is described followed by verification using simple structures. Then extended sensitivity analyses for operational deflection shape, relative motion, transmitted force, servo system and acoustics are discussed with practical applications.

\section{Theoretical background and verification}

\subsection{Natural frequency sensitivity}

When the shift of one natural frequency of structure, $f_{i}$, at the $i$ th mode shape, with respect to the design parameter, $P_{j}$ at point $j$ is considered, sensitivity can be derived as follows. The equation of motion at the $i$ th mode shape, $\{\phi\}_{i}$, normalized to unit modal mass is written, without damping for simplicity, as

$$
[K]\{\phi\}_{i}=\lambda_{i}[M]\{\phi\}_{i},
$$

where $\lambda_{i}\left(=\left(2 \pi f_{i}\right)^{2}\right)$ is the $i$ th eigenvalue and $[M]$ and $[K]$ are the mass and stiffness matrix respectively. By differentiating each term with respect to $P_{j}$, the sensitivity is finally expressed as

$$
\frac{\partial \lambda_{i}}{\partial P_{j}}=\{\phi\}_{i}^{T}\left(\frac{\partial[K]}{\partial P_{j}}-\lambda_{i} \frac{\partial[M]}{\partial P_{j}}\right)\{\phi\}_{i} .
$$

If lumped mass distribution and scalar springs are assumed, $\partial[M] / \partial P_{j}$ and $\partial[K] / \partial P_{j}$ become very simple, and most elements are zero except those related with point $j$. Equation (2) can be calculated straightaway either numerically using FEM or an experimental modal model.

To verify this sensitivity analysis experimentally, a structure of computer disk shown in figure 1 is used. The modal testing is carried out and typical FRF and one mode shape are also shown in the figure.

Based on the modal parameters measured, the natural frequency sensitivities for mode 4 are calculated as shown in figure 2, using a sensitivity map which indicates the sensitivity level when adding unit mass at each measurement point.

When the most sensitive point, point 15 in this case, is selected, the FRF is changed, as also shown in the figure, due to the mass modification, while in the case of the moderate

(a)

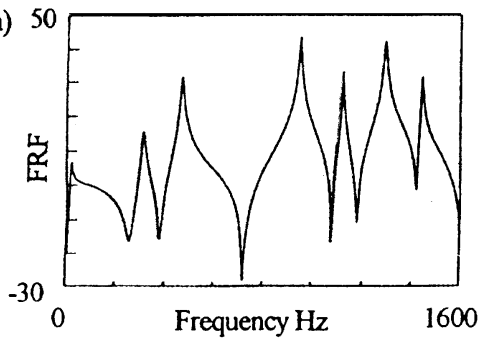

(b)

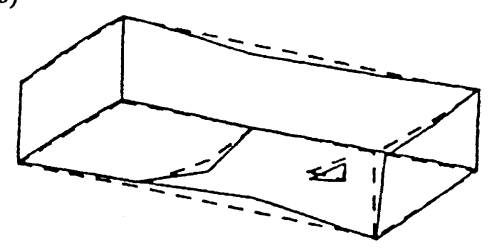

MODE $4 \quad 1119.35 \mathrm{~Hz}$

Figure 1. (a) FRF, and (b) mode shape of computer disk structure. 


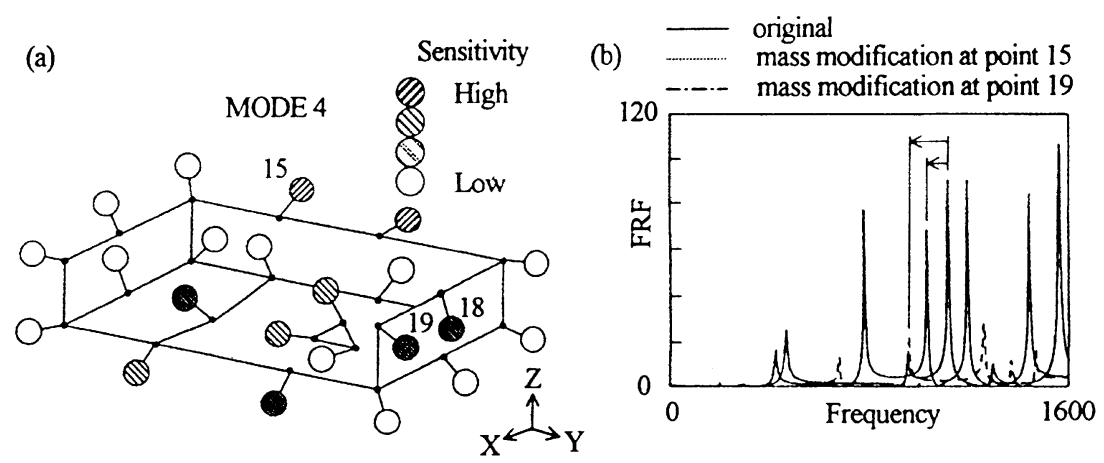

Figure 2. (a) Natural frequency sensitivity map due to mass modification, and (b) the change of FRF.

sensitive point 19 , the natural frequency under consideration is not shifted much compared to point 15 as predicted.

\subsection{Mode shape sensitivity}

In case of natural frequency sensitivity analysis, the shift of the natural frequency under consideration is our only concern. If the mode shape or the FRF at the natural frequency is the primary concern, the mode shape sensitivity should be taken into account.

An assumption is made where the mode shape sensitivity can be expressed by superposing the unmodified mode shapes with appropriate scaling $a_{i r}$ as,

$$
\frac{\partial\{\phi\}_{i}}{\partial P_{j}}=\sum_{r=1}^{n} a_{i r}\{\phi\}_{r}
$$

where $n$ is the number of mode shapes.

By substituting (3) into (1), the final expression for scaling can be obtained as

$$
\begin{aligned}
& a_{i r}=\frac{1}{\lambda_{r}-\lambda_{i}}\{\phi\}_{r}^{T}\left(\lambda_{i} \frac{\partial[M]}{\partial P_{j}}-\frac{\partial[K]}{\partial P_{j}}\right)\{\phi\}_{i} \quad(i \neq r), \\
& a_{i i}=-\frac{1}{2}\{\phi\}_{i}^{T} \frac{\partial[M]}{\partial P_{j}}\{\phi\}_{i} .
\end{aligned}
$$

Compared with (2) for natural frequency sensitivity, which needs only the associated natural frequency $f_{i}$ and mode shape $\{\phi\}_{i}$, mode shape sensitivity requires all the natural frequencies and mode shapes. From the experimental point of view, therefore the error due to mode truncation, that is the number of mode shapes available, should be assessed in order to conduct proper mode shape sensitivity analysis.

The computer disk structure is again used to verify this analysis. Figure 3 shows the mode shape sensitivity map at point 5 in the $Z$ direction for mode 4 due to mass modification. When the most sensitive point, point 15 in this case, is selected, the FRF is changed, as shown in the figure, due to the mass modification. In case of the moderately sensitive point 8, the FRF amplitude under consideration is not changed much when compared to point 15 as predicted. 

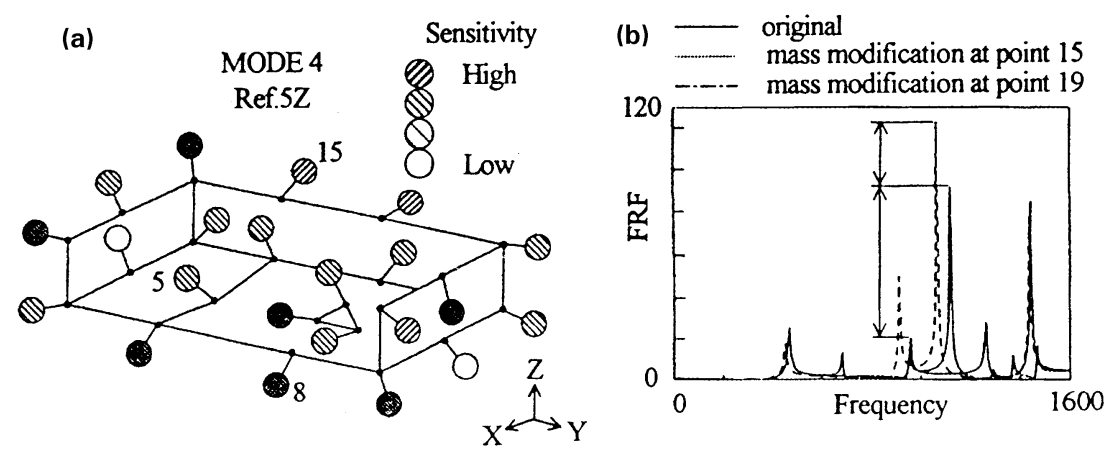

Figure 3. (a) Mode shape sensitivity map due to mass modification, and (b) the change of FRF.

As seen in the figure, the change of FRF amplitude, whether it increases or decreases, can be properly predicted. However the shift of natural frequency of the associated mode shape is ignored.

\section{Extended sensitivity analysis}

\subsection{Operational deflection shape sensitivity}

The above mentioned natural frequency and mode shape sensitivity are useful to solve a dynamic problem, the so-called resonance problem where only one mode shape is dominant and the number and the points of action of the inputs on the structure during operation are not important.

On the contrary, a dynamic problem at off resonance is very much dependent on the inputs and is referred to as the operational deflection shape (ODS), which is a combination of mode shapes. The coefficient of each mode shape for the combination is defined as a modal coordinate and is a function of where and how many inputs are acting at the frequency. In order to identify the inputs, force identification can be used based on the system model, FRFs in measurement, and the responses under operating conditions (Okubo 1977).

The ODS sensitivity can be thus calculated as below (note that only mass modification is considered for simplicity and $\partial[K] / \partial p_{j}=0$ ),

$$
\begin{aligned}
& \frac{\partial\{X\}}{\partial P_{j}}=\left[\sum _ { r = 1 } ^ { n } \left(\frac{\{\phi\}_{r}\left(\partial\{\phi\}_{r}^{T} / \partial P_{j}\right)+\left(\partial\{\phi\}_{r} / \partial P_{j}\right)\{\phi\}_{r}^{T}}{\left(-\omega^{2}+\lambda_{r}^{2}\right)}\right.\right. \\
&\left.\left.+\frac{\omega^{2}\{\phi\}_{r}^{T}\left(\partial[M] / \partial P_{j}\right)\{\phi\}_{r}\{\phi\}_{r}^{T}}{\left(-\omega^{2}+\lambda_{r}^{2}\right)^{2}}\right)\right]\{F\}, \\
&\{F\}=\left([\Phi][\Phi]^{T}\right)^{-1}[\phi]\left(-\omega^{2}\left[{ }^{\prime} m_{\downarrow}\right]+\left[k^{\prime}\right]\right)\left([\Phi]^{T}[\Phi]\right)^{-1}[\Phi]^{T}\{\bar{X}\},
\end{aligned}
$$

where $\{X\}$ is ODS at angular frequency $\omega$ under consideration, $[\Phi]$ is the mode shape matrix, $m$ and $k$ are the modal mass and stiffness and $\{\bar{X}\}$ is the response at $\omega$ under operating conditions.

This sensitivity analysis is applied to a laser beam printer as shown in figure 4, where the polygon mirror and chart driving motor are the main sources for generating vibrations. The 


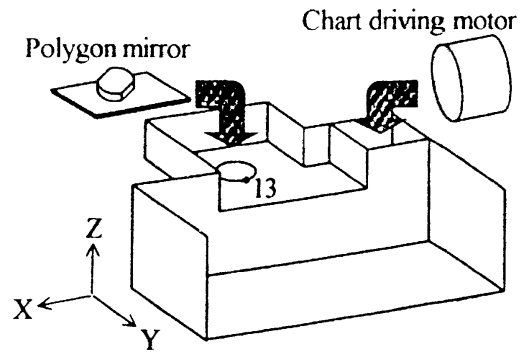

Figure 4. ODS sensitivity analysis on laser beam printer.

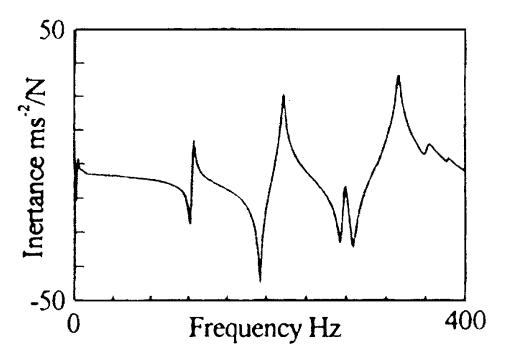

Figure 5. FRF of laser beam printer.

FRF is measured and shown in figure 5. Then the vibrations of the chart driving motor are analysed as shown in figure 6 under free-free condition removed from the structure, which reveals the fundamental frequency with harmonics as a rotating device. Compared with the FRF, the natural frequencies are so designed as to not coincide with these forcing frequencies. Nevertheless, the vibration at $154 \mathrm{~Hz}$ still needs further improvement since even a low level of vibration may cause trouble for such a high precision device.

The ODS sensitivity at $154 \mathrm{~Hz}$ due to mass modification is applied and the sensitivity map is calculated as shown in figure 7 to determine the most effective point for suppressing the vibration (Kishi \& Okubo 1993).

\subsection{Relative motion sensitivity}

When dynamic improvement is under consideration, not only the absolute motion but also relative motion should be analysed. For example, a microscope for inspection of large scale integration circuit demands higher magnification, which is influenced by the relative motion between the objective lens and the stage in the horizontal plane as shown in figure 8. In the figure the relative FRF is also shown which suggests the necessity of vibration isolation from a base for lower frequency range.

The relative motion sensitivity between points $a$ and $b$ can be derived by differentiating the combination of mode shapes with modal coordinate $\gamma$ as follows,

$$
\frac{\partial\left(X_{a}-X_{b}\right)}{\partial P_{j}}=\gamma_{1} \frac{\partial\left(\phi_{a}-\phi_{b}\right)_{1}}{\partial P_{j}}+\gamma_{2} \frac{\partial\left(\phi_{a}-\phi_{b}\right)_{2}}{\partial P_{j}}+\cdots+\gamma_{n} \frac{\partial\left(\phi_{a}-\phi_{b}\right)_{n}}{\partial P_{j}} .
$$

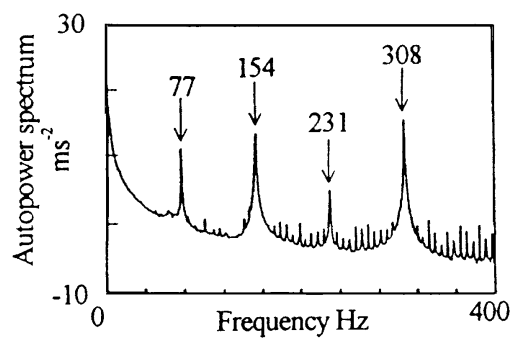

Figure 6. Vibration of chart driving motor.

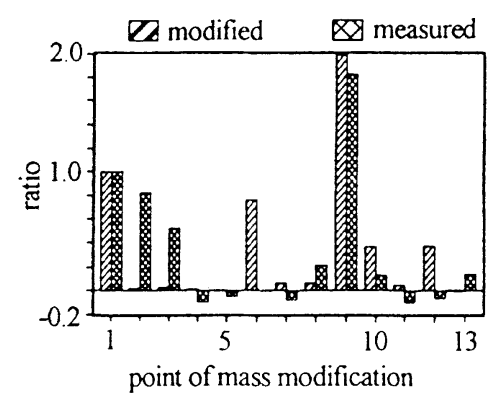

Figure 7. ODS sensitivity map due to mass modification. 


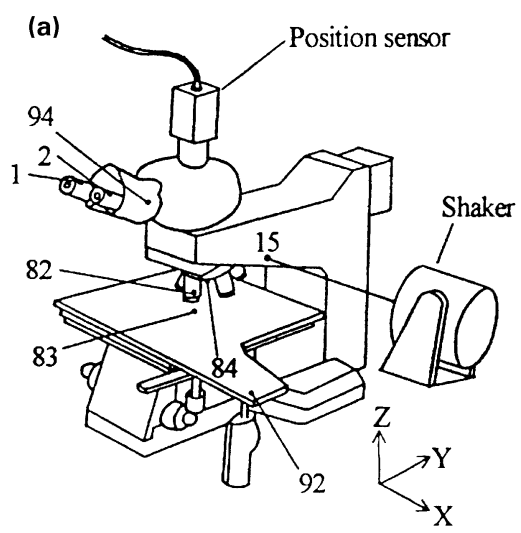

(b)

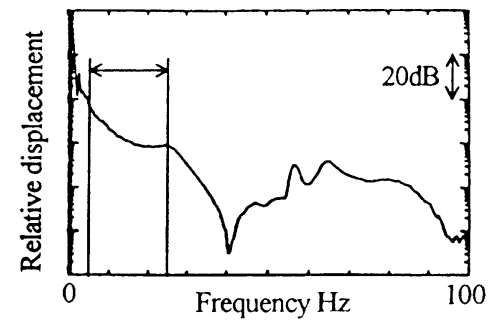

Figure 8. (a) Microscope under test, and (b) relative FRF.

This relative motion sensitivity analysis is applied to the microscope for the frequency range 5 to $25 \mathrm{~Hz}$ as shown in figure $8 \mathrm{~b}$ which is lower than the first elastic mode shape. Below the first mode shape, one might think that the first mode shape is dominant, which is not always true in case of relative motion, and apply conventional sensitivity analysis. As a result point 94 is inferred to be the most sensitive with respect to mass modification as shown in figure 9, while relative motion sensitivity analysis gives point 84 as the most sensitive one. Figure 10 shows the comparison of the relative motion before and after $1 \%$ mass addition at each point. This confirms that only relative sensitivity analysis can provide proper isolation (Toi et al 1997).

\subsection{Transmitted force sensitivity}

In many cases, a machine consists of a main component, which contains a vibration source and a sub-component without a vibration source, these being connected through a few connecting points. Under these circumstances, the transmitted force from the main component to the sub-component should be taken into consideration for effective reduction of vibration and vibration-induced noise of the sub-component. This is because conventional
$80-100 \%$
$60-80 \%$
(3) $40-60 \%$
(3) $20-40 \%$
○ $0-20 \%$
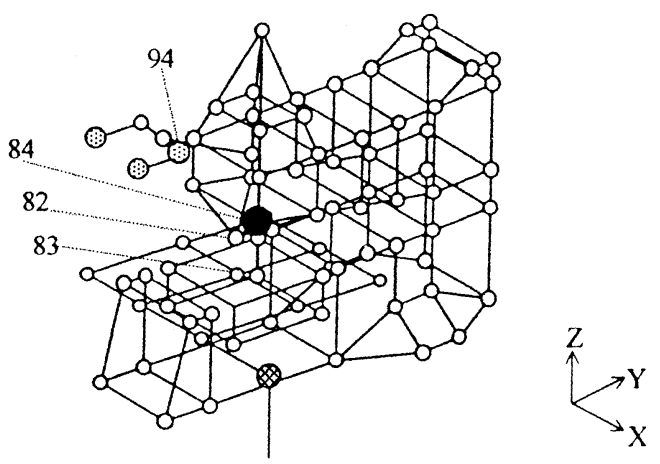

Figure 9. Comparison of conventional and relative motion sensitivity map due to mass modification. 


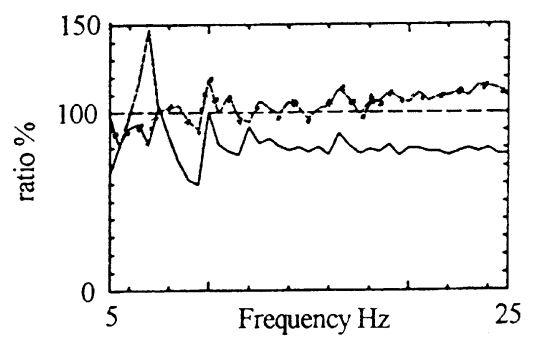

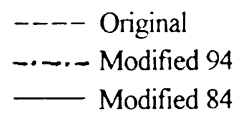

Figure 10. Reduction of relative motion due to mass modification.

sensitivity analysis can determine appropriate modification points in order to suppress the vibration at one point in one direction as previously mentioned. Here, a new sensitivity analysis is developed so as to take into account the transmitted force as follows (Okubo et al 1996).

As shown in figure 11 , an input force $F_{1}$ acts at the main component 1 , which is independent of the modification, and transmits it to the sub-component 2 through points 2 and 3. Then (for simplicity only mass modification $\Delta m_{c}$ at point $c$ is discussed) the modified FRF between input point $b$ and response point $a, H_{a b}^{*}$ can be written by direct use of FRF as

$$
H_{a b}^{*}=H_{a b}-H_{a c} H_{c b} /\left(H_{c c}+\frac{1}{\Delta m_{c}}\right) .
$$

Therefore the transmitted force sensitivity can be expressed as a ratio as follows,

$$
\frac{F_{2}}{F_{1}}=\frac{o\left(\Delta m_{c}\right)^{2}+p\left(\Delta m_{c}\right)+q}{r\left(\Delta m_{c}\right)^{2}+s\left(\Delta m_{c}\right)+t},
$$

where coefficients, $o, p, q, r, s$ and $t$ are calculated from the modified FRFs.

This transmitted force sensitivity analysis is applied to a motorbike shown in figure 12a, where the main frame is excited by an input and the muffler component is supposed to generate vibration and vibration-induced noise due to transmitted forces from the main

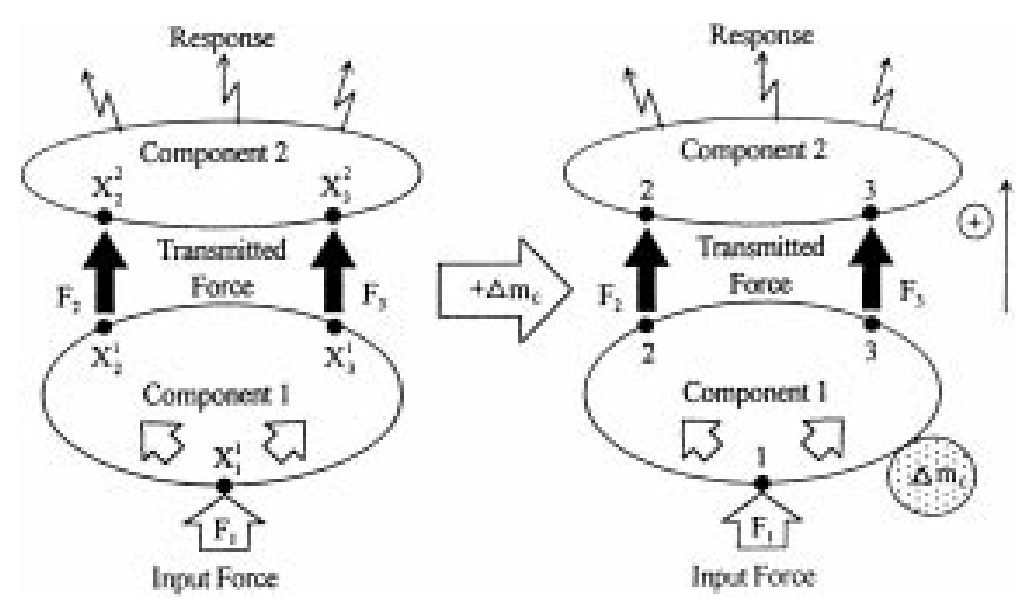

Figure 11. Transmitted force sensitivity analysis. 

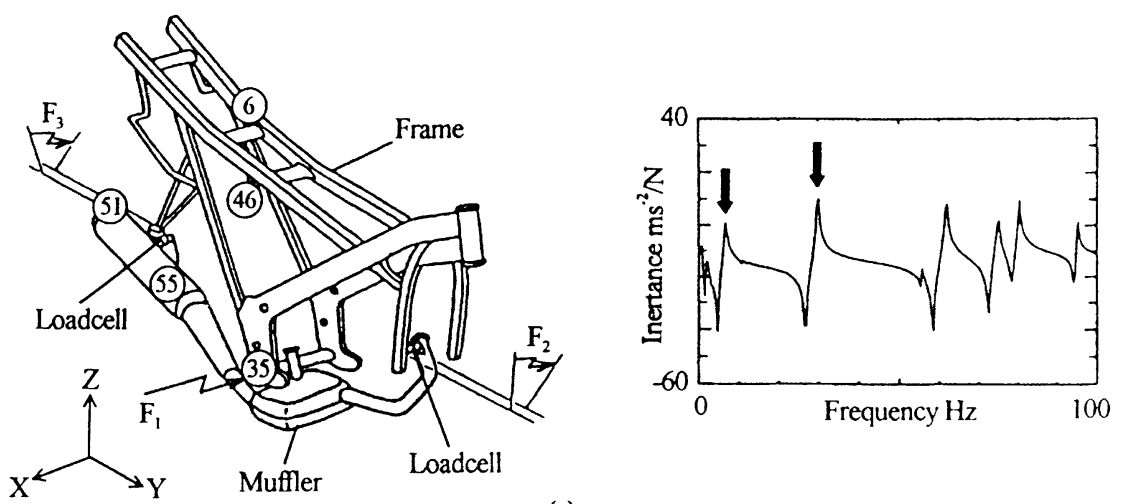

(a)

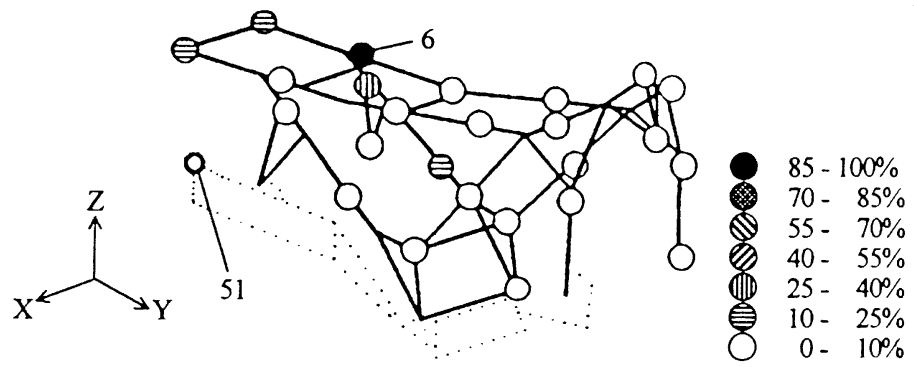

(b)

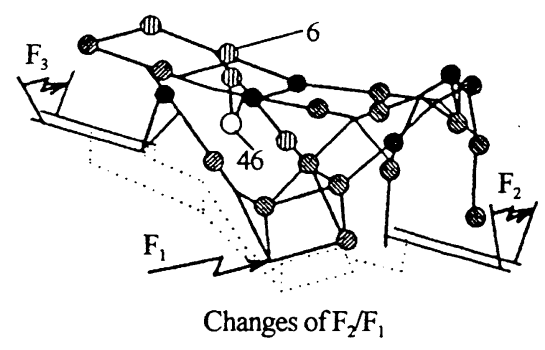<smiles>[X]C([X])[X]</smiles>

(c)

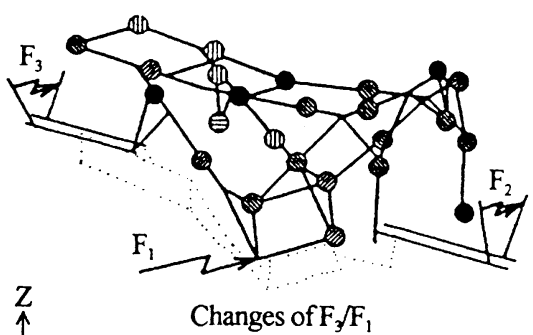

120
$105-120 \%$
$95-105 \%$
$80-95 \%$
$65-80 \%$
$50-65 \%$
$-50 \%$

Figure 12. Motorbike frame under test (a), and comparison of sensitivity map by conventional (b) and transmitted force (c).

frame through two connecting points. To reduce the vibration of the muffler, conventional sensitivity analysis is used to suppress the vibration at one point of the muffler and the sensitivity map is obtained as shown in figure $12 \mathrm{~b}$.

On the other hand, transmitted force sensitivity analysis maps are calculated as shown in figure $12 \mathrm{c}$ and the appropriate point for mass addition can be determined in order to reduce the two transmitted forces. 


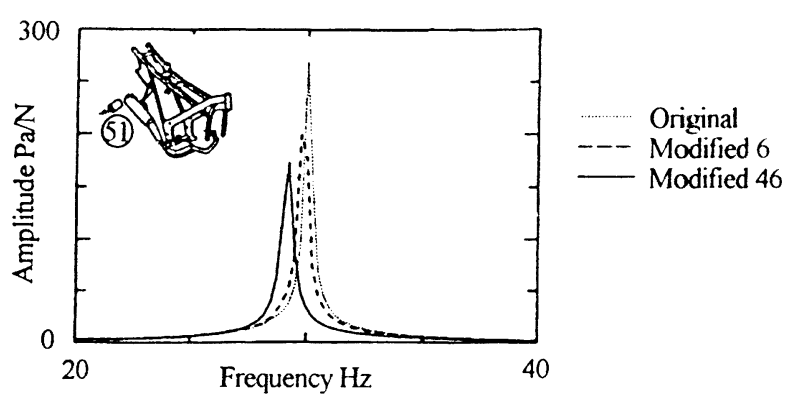

Figure 13. Comparison of reduction of vibration induced noise by conventional and transmitted force sensitivity.

Finally, vibration-induced noise is measured before and after the mass modification as shown in figure 13, which confirms that transmitted force sensitivity can provide the most effective reduction compared with conventional sensitivity analysis.

\subsection{Servo sensitivity}

Modern machines have become so smart as to equip a servo system called mechatronics to control unpleasant vibration and noise. Therefore, the sensitivity analysis should be extended to involve not only mechanical elements but also electrical elements of the servo

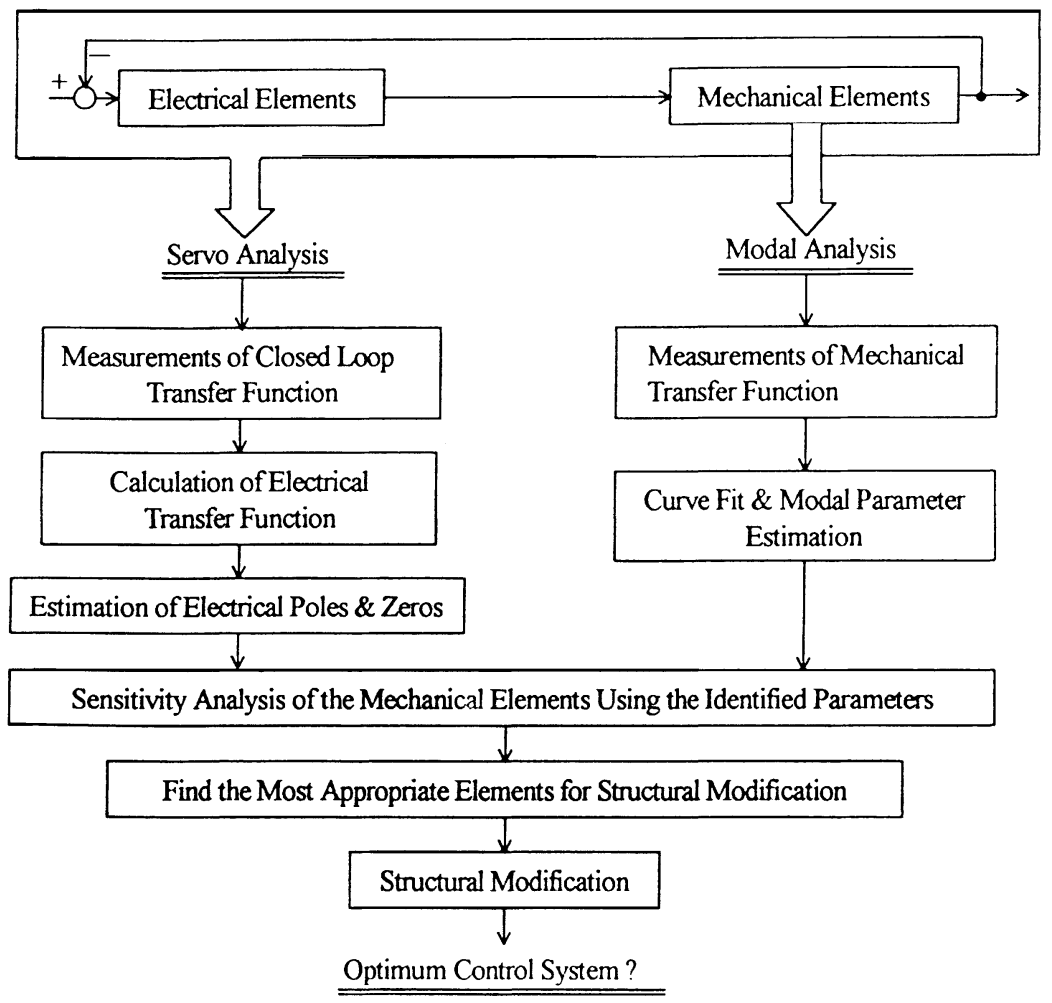

Figure 14. Servo sensitivity analysis with mechanical and electrical elements. 

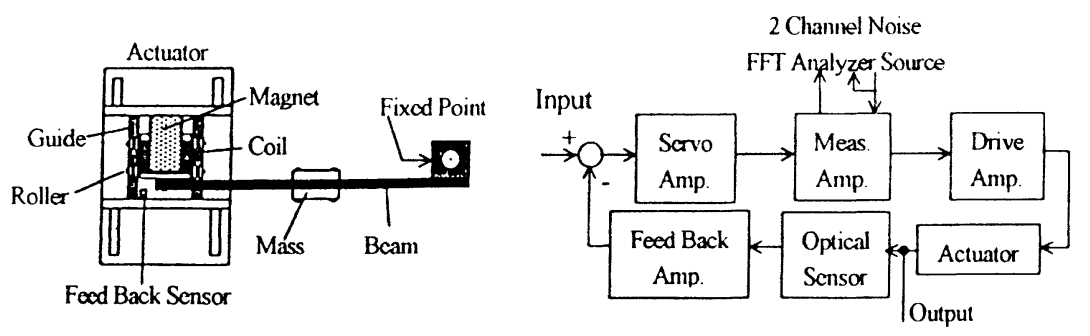

Figure 15. Mechatronics system under test.

system. As shown in figure 14, the mechanical transfer function can be measured with common modal testing while the servo system is off, and on the other hand the closed loop transfer function can also be measured by the so-called servo measurement. The electrical transfer function can then be identified and used to extract poles and zeros. Based on these parameters, servo sensitivity can be defined as the ratio of change of closed loop transfer function $T(s)$ to design parameters, as

$$
S_{p j}=\frac{\partial T(s) / T(s)}{\partial P_{j} / P_{j}} .
$$

A simple mechatronics structure to control the position of the moving coil supported by a beam is used to verify this analysis, as shown in figure 15 , which also illustrates the block diagram of servo measurement to measure the closed loop transfer function. In figure 16, the measured mechanical transfer function by common modal testing and the closed loop transfer function by servo measurement are shown together with the identified electrical transfer function.

Servo sensitivity analysis then provides the change of closed loop transfer function due to mass modification, which is plotted as a function of frequency as shown in figure 17. The figure also shows the measured change when the mass is actually added and good agreement between the predicted and measured is observed (Okubo \& Yamamoto 1987).

\subsection{Acoustic sensitivity}

Noise generated by a machine becomes a critical measure when a consumer purchases the machine. Especially in case of vibration-induced noise, acoustic modal testing is often utilized to investigate the acoustic behaviour of the structure, where a microphone is used

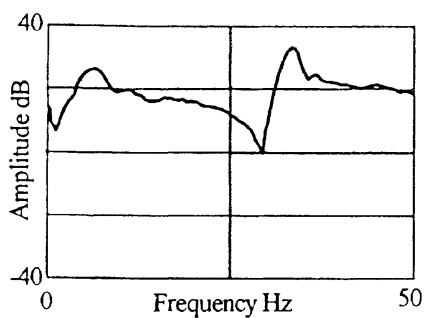

(a)

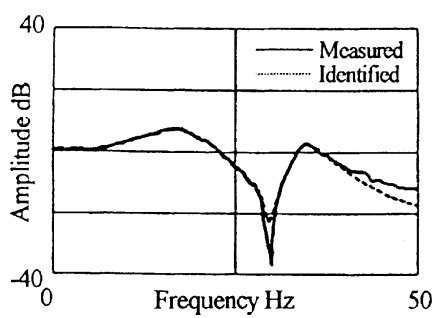

(b)

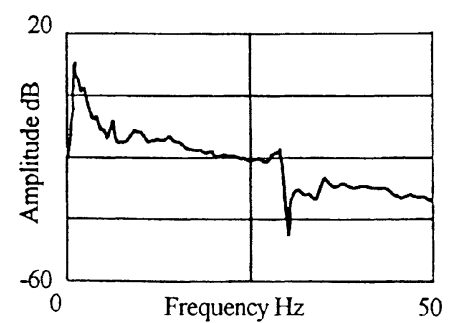

(c)

Figure 16. (a) Mechanical, (b) closed loop, and (c) electrical transfer functions. 


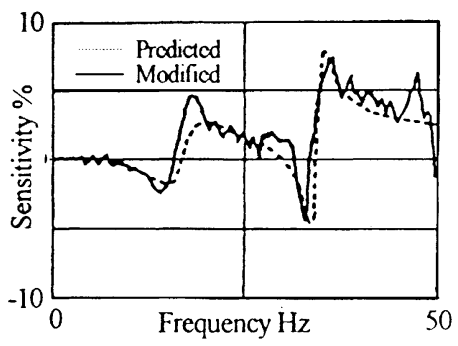

Figure 17. Comparison of the amplitude servo sensitivity due to mass modification measured and predicted.

to detect the acoustic pressure. The acoustic FRF between the acoustic pressure at observation point $P$ and the input force $F_{i}$ at point $i$ can be written under the assumption of point source model as

$$
\begin{aligned}
& H_{i}=\frac{P}{F_{i}}=\frac{\rho \omega^{2}}{2 \pi} \sum_{r=1}^{n}\left\{\frac{\phi_{i r} \psi_{r}}{j \omega-\lambda_{r}}+\frac{\phi_{i r}^{*} \psi_{r}^{*}}{j \omega-\lambda_{r}^{*}}\right\}, \\
& \psi_{r}=\sum_{k=1}^{m} \phi_{k r} \Delta S_{k} \exp \left(-j k R_{k}\right) / R_{k}, \\
& \lambda_{r}=-\sigma_{r}+j \omega_{r},
\end{aligned}
$$

where $\rho$ is the air density, $k$ the wave number, $\omega_{r}$ the $r$ th angular natural frequency, $\sigma_{r}$ the $r$ th damping factor, $\phi_{i r}$ the $i$ th component of the $r$ th vibration mode shape, $\Delta S_{k}$ the equivalent area at point $k, R_{k}$ the distance between the microphone and the point $k$ on the structure, $n$ the number of mode shapes, $m$ the number of measurement points on the structure and $*$ the conjugate term.

The acoustic sensitivity with respect to design parameter, therefore, can be derived by differentiating (11). Figure 18 shows a typical setup for acoustic modal testing of a 4
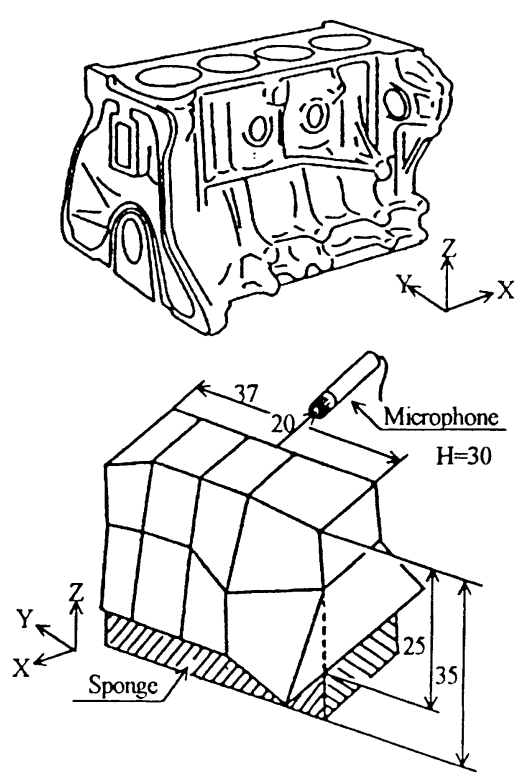

Figure 18. Automobile engine block under acoustic modal testing. 


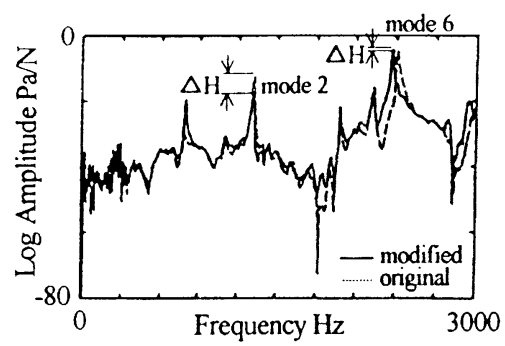

Figure 19. Acoustic FRF before and after mass modification.

cylinder automobile engine block. The measured acoustic FRF is shown in figure 19, where the peaks coincide with the natural frequencies. Also acoustic FRF after mass modification is shown. In figure 20, the predicted acoustic sensitivity map is shown to suggest the sensitive points where a small mass achieves a large noise reduction. For comparison the measured map is also shown when a mass is actually added at each point and the change is evaluated, which agrees well with the prediction by the acoustic sensitivity analysis (Okubo $\&$ Masuda 1990).

\section{Conclusions}

Natural frequency sensitivity and mode shape sensitivity are described and applied to a simple structure for verification of its effectiveness. By making good use of these analyses, one can determine appropriate points to suppress the vibration by small modifications.

Sensitivity analysis can be extended to deal with operational deflection shape, relative motion, transmitted force, servo system and acoustics. Practical examples are given to illustrate the results of such extended application.

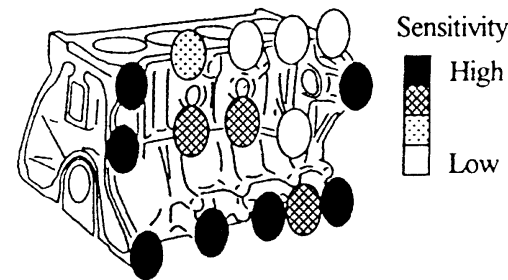

Acoustic Predicted $1317 \mathrm{~Hz}$
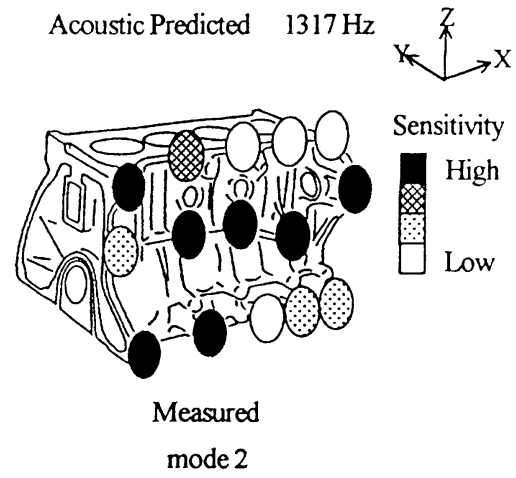

Figure 20. Acoustic sensitivity map, predicted and measured. 


\section{References}

Kishi K, Okubo N 1993 Sensitivity analysis on reduction of operational vibration for laser printer. JSPE Spring Meeting pp. 753-754 (in Japanese)

Okubo N 1997 Force identification and its application. Proceedings of the International Winter School on Optimum Dynamic Design (Delhi: Allied Publishers)

Okubo N, Masuda K 1990 Acoustic sensitivity analysis based on the results of acoustic modal testing. Proc. 8th Int. Modal Anal. Conf.

Okubo N, Yamamoto H 1987 Servo sensitivity - extended structural sensitivity analysis for an electro-mechanical control system Proc. 5th Int. Modal Anal. Conf.

Okubo N, Toi T, Aoyama K 1996 Prediction of transmitted force between components under operating condition for reduction of vibration and noise. Proc. 14th Int. Modal Anal. Conf.

Toi T, Murase Y, Okobo N 1997 Reduction of vibration for main functional part in precision device based on sensitivity analysis with respect to relative motion. J. Jpn. Soc. Precision Eng. 63(1) 